\title{
AtrapaelTigre.com: enlisting citizen-scientists in the war on tiger mosquitoes
}

\author{
Aitana Oltra*, John R.B. Palmer ${ }^{\dagger}$ and \\ Frederic Bartumeus ${ }^{*,+, \neq}$
}

*CEAB-CSIC, Cala Sant Francesc 14, 17300 Blanes, Spain, aoltra@ceab.csic.es

${ }^{\dagger}$ CREAF, Cerdanyola del Vallès, 08193 Barcelona, Spain

¥ICREA, Pg. Lluís Companys 23, 08010 Barcelona, Spain

\begin{abstract}
This chapter describes AtrapaelTigre.com, a citizen science project focusing on the Asian tiger mosquito in Spain. Commonly known for its aggressive biting during the day, the tiger mosquito represents a global environmental problem. It is an invasive species and a vector for dengue, chikungunya and other diseases, making it a serious public health risk. It is also an everyday nuisance and a threat to tourism and related industries. The management of invasive species, and particularly disease vectors, requires integrated programs that combine public communication and education with research, surveillance and control. AtrapaelTigre.com aims at achieving this by engaging citizen scientists to raise awareness and collect data on tiger mosquito adults and their breeding sites with a smartphone app (Tigatrapp) and a multi-proxy data validation system that combines expert, crowd, and app-user input. Lessons learned during the first year of implementation in Spain, in 2014, have guided our current strategies with respect to both tiger mosquitoes and the formal integration of citizen
\end{abstract}

\section{How to cite this book chapter:}

Oltra, A, Palmer, J R B and Bartumeus, F. 2016. AtrapaelTigre.com: enlisting citizen-scientists in the war on tiger mosquitoes. In: Capineri, C, Haklay, M, Huang, H, Antoniou, V, Kettunen, J, Ostermann, F and Purves, R. (eds.) European Handbook of Crowdsourced Geographic Information, Pp. 295-308. London: Ubiquity Press. DOI: http://dx.doi.org/10.5334/bax.v. License: CC-BY 4.0. 
science into the research, surveillance and control of invasive species and disease vectors generally. We address the challenges of implementing such frameworks and discuss their fitness for use in public health systems. The goal of AtrapaelTigre.com is not only to enhance participation and raise awareness, but also to promote novel research and a more informed and cost-effective management of the tiger mosquito across Spain.

\section{Keywords}

citizen science, volunteered geographic information, invasive species, disease vectors, Asian tiger mosquito

\section{Introduction}

AtrapaelTigre.com ('Catch-the-Tiger') is a citizen science project with a Volunteered Geographic Information (VGI) (Goodchild 2007) component that enlists ordinary people in the research, surveillance and control of Asian tiger mosquitoes (Aedes albopictus) (Skuse 1894) in Spain. The tiger mosquito is an invasive species from Southeast Asia that has spread worldwide and become common in developed landscapes (Hawley 1988). It is well known for biting aggressively during the day, and importantly, it is a vector of such diseases as dengue and chikungunya (Paupy et al. 2009). The species is relatively easy to recognize from its behavior and appearance, and it was first detected in Spain in 2004 (Aranda et al. 2006). The tiger mosquito is now established along the Spanish Mediterranean coast (Alarcón-Elbal et al. 2014), where it threatens public health and degrades the quality of life, while also harming the tourism sector (Roiz et al. 2007), which peaks in the summer, just when the species is most active.

The efficacy of public management programs is limited because tiger mosquitoes breed not only in public spaces, but also in small water containers in private areas, such as the plates people place under their flower pots on balconies and patios. Removing the water from these containers prevents the development of larvae and may significantly reduce the presence of adults at a given place. This can be especially effective, since the lifetime dispersal range of the species is only around $600 \mathrm{~m}$ (Hawley 1988). Therefore, awareness-raising and education campaigns are key tools for control programs. This, combined with the ease with which tiger mosquitoes can be identified and the extent to which they are often well known to the communities in which they are prevalent, makes the species a good target for citizen science. A number of projects have recently begun using public participation to monitor mosquitoes elsewhere (e.g. Mückenatlas in Germany, iMoustique in France) (Kampen et al. 2015). Although invasive species are common citizen science targets (e.g. Dickinson 
et al. 2010), it is much less common to specifically target disease vectors like the tiger mosquito that affect humans.

AtrapaelTigre.com has two specific objectives: (1) to explore new methodologies for acquiring data for tiger mosquito research, surveillance and control through public participation, and (2) to raise public awareness and promote household control actions. The project was initiated in 2013 as a pilot in a small region of Spain with a limited target group of participants. The know-how and network acquired served to extend the approach in 2014 to the whole territory of Spain. Here we present the existing system (data collection, validation and visualization) and the lessons learned in 2014, during the first year of implementation in Spain. We also placed the project and future challenges in a broader conceptual framework useful for other citizen science projects targeting invasive species and disease vectors.

\section{Data collection and validation approach}

Data collection is done using the smartphone app Tigatrapp, available on Google Play and iTunes. Tigatrapp is free and open source software, ${ }^{1}$ and it may be redistributed or modified under the GNU General Public License (version 3). Participation is anonymous, but participants must consent first to the privacy policy and terms of use. With Tigatrapp, ordinary people can collect and send geolocalized reports of tiger mosquitoes and their breeding sites (Figure 1). To do this, they need to learn how to identify the tiger mosquito (including basic taxonomy and life cycle), and this information is provided by the app and the project website, as well as in workshops and talks organized throughout the mosquito season. Tigatrapp reports include (Figure 1): i) location, obtained by the app directly from the device's GPS receiver or its network connections or from the user selecting the location on a map, ii) key taxonomic traits of the reported mosquito or characteristics of the reported breeding site based on a small survey (i.e. user level validation), iii) photographs (compulsory for breeding sites but optional for adult mosquitoes, which are often hard to photograph), and iv) optional complementary notes.

The app also collects 5 randomly-timed, anonymous samples of user locations on a daily basis (although users have the option to switch this feature off). This background location system (Figure 1) is used to estimate sampling effort and territorial exposure, making it possible to adjust the report analysis for the fact that users are not randomly distributed across the landscape. In other words, the background location system helps in identifying the extent to which reports from a given area are being driven by the density of users, the density of mosquitoes, or both. In order to protect privacy, all background location information is masked on users' devices by placing locations on a predetermined

\footnotetext{
${ }^{1}$ https://github.com/MoveLab.
} 


\begin{tabular}{|c|c|}
\hline \multicolumn{2}{|c|}{ DATA COLLECTION APP TIGATRAPP } \\
\hline \multicolumn{2}{|c|}{ REPORTS } \\
\hline MOSQUITOES & BREEDING SITES \\
\hline $\begin{array}{c}\text { Location } \\
\text { Taxonomic survey } \\
\text { Photographs (optional) } \\
\text { Notes (optional) }\end{array}$ & $\begin{array}{c}\text { Location } \\
\text { Sites survey }\end{array}$ \\
\hline OTHER DATA & $\begin{array}{c}\text { Photographs } \\
\text { Notes (optional) }\end{array}$ \\
\hline BACKGROUND LOCATION & MISSIONS \\
\hline $\begin{array}{c}\text { Approximate location 5 times/day } \\
\text { (optional) }\end{array}$ & $\begin{array}{c}\text { Variable information } \\
\text { sent/collected to/from a specific } \\
\text { region (optional) }\end{array}$ \\
\hline
\end{tabular}

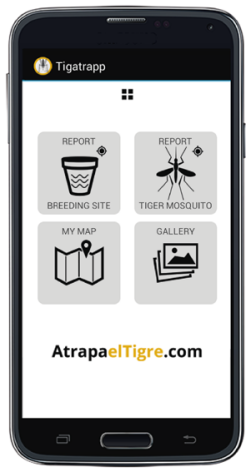

Figure 1: Data collected with Tigatrapp. Background location and missions are not yet implemented in the iOS version of the app due to financial constraints.

grid of 0.05 degrees latitude and longitude. Only the grid cell identifier is transmitted from the device to the server, making it impossible to determine the actual location within the cell. In addition, these locations are identified only by a code that is randomly assigned on the user's device, without any additional information or any way to link the location to a given user's reports.

Finally, the app incorporates the concept of missions (Figure 1), which are extra voluntary activities, notes or surveys that are sent as an incoming notification to Tigatrapp. These can be set to appear on the devices of all users or only those in a given area. Missions allow the implementation of extra activities on the go, and direct communication with participants, according to specific needs.

The two types of reports (mosquito adults and breeding sites) and the sampling effort (covered area) can be visualized on a webmap embedded in the project website. The coverage map is also a useful tool for increasing user engagement, as people are able to see the extent to which the app is being used across Spain and, indeed, throughout the world. Raw data is also available to some scientists (even outside the project) and to some public administrations responsible for tiger mosquito control and additional data will be made available to the public through public-access repositories and other means in the future.

To validate reports, a multi-proxy system that combines expert, crowd and app-user validation has been implemented (Figure 2). Expert and crowd validation are based on the analysis of report photographs and are done using two different on-line platforms: a) a custom-built platform for experts and b) Crowdcrafting.org (Tigafotos project) for the public. App-user validation is based on the user's responses to the survey contained in each submitted report. For expert validation, the experts analyze the photographs to classify each report into one of five categories (Figure 2) based on the assessed probability of its being accurate (i.e. the probability that the user actually observed a tiger mosquito or a tiger mosquito breeding site). Expert validation is done before publishing report photographs on the webmap and thus, also serves to filter 
out pictures that are sensitive (e.g. bites on people's bodies), include personal information (e.g. whole body or face pictures), or are unrelated to the project (although there have been few of these). Experts can also decide to publish the app-user's note (if available) or to add their own note in the report popup on the webmap. Expert validation results are used as the main filter and

\begin{tabular}{|c|c|c|c|c|}
\hline \multicolumn{5}{|c|}{ USER VALIDATION (App report survey) } \\
\hline \multicolumn{5}{|c|}{$\begin{array}{c}\text { NEW TIGATRAPP } \\
\text { REPORT }\end{array} \rightarrow$ Photographs? } \\
\hline \multicolumn{5}{|c|}{$\sqrt{ }$} \\
\hline \multicolumn{2}{|c|}{ CROWD VALIDATION (Crowdcrafting) } & \multicolumn{3}{|c|}{ EXPERT VALIDATION (own platform) } \\
\hline \multicolumn{2}{|c|}{$\begin{array}{l}\text { Assigns a category based on the } \\
\text { opinion of the crowd }\end{array}$} & \multicolumn{3}{|c|}{$\begin{array}{l}\text { Assigns a category based on the } \\
\text { opinion of several experts }\end{array}$} \\
\hline & I & & & \\
\hline Report & classified & $\begin{array}{l}\text { PUBLIC } \\
\text { WEBMAP }\end{array}$ & Report not & classified \\
\hline \\
\hline \multirow{2}{*}{\multicolumn{5}{|c|}{ EXAMPLE: CATEGORY CLASSIFICATION FOR MOSQUITO REPORTS }} \\
\hline & & & & \\
\hline $\begin{array}{c}\text { CONFIRMED } \\
\text { TIGER } \\
\text { MOSQUITO }\end{array}$ & $\begin{array}{l}\text { POSSIBLE } \\
\text { TIGER } \\
\text { MOSQUITO }\end{array}$ & UNKNOWN & $\begin{array}{l}\text { POSSIBLE } \\
\text { OTHER } \\
\text { SPECIES }\end{array}$ & $\begin{array}{l}\text { OTHER } \\
\text { SPECIES }\end{array}$ \\
\hline $\begin{array}{l}\text { Very likely } \\
\text { tiger mosquito }\end{array}$ & $\begin{array}{l}\text { Could be tiger } \\
\text { mosquito. } \\
\text { Cannot be } \\
\text { assured reliably }\end{array}$ & $\begin{array}{c}\text { Not possible } \\
\text { to assign any } \\
\text { other } \\
\text { category }\end{array}$ & $\begin{array}{l}\text { Could be } \\
\text { another species. } \\
\text { Cannot be } \\
\text { assured reliably }\end{array}$ & $\begin{array}{c}\text { Almost } \\
\text { definitely not } \\
\text { tiger } \\
\text { mosquito }\end{array}$ \\
\hline $\begin{array}{l}\text { Reports with a } \\
\text { photograph } \\
\text { that looks like } \\
\text { a tiger } \\
\text { mosquito. The } \\
\text { white stripe on } \\
\text { its head and } \\
\text { thorax, unique } \\
\text { to this } \\
\text { species, is } \\
\text { properly } \\
\text { recognized }\end{array}$ & $\begin{array}{l}\text { Reports with a } \\
\text { photograph that } \\
\text { looks like a } \\
\text { tiger mosquito. } \\
\text { The white } \\
\text { stripe cannot } \\
\text { be properly } \\
\text { recognized. } \\
\text { These could } \\
\text { also describe } \\
\text { other similar } \\
\text { species }\end{array}$ & $\begin{array}{l}\text { Reports with } \\
\text { photographs } \\
\text { that are not } \\
\text { informative } \\
\text { enough (e.g. } \\
\text { blurry, } \\
\text { overexposed) } \\
\text { for a better } \\
\text { decision }\end{array}$ & $\begin{array}{l}\text { Reports with a } \\
\text { photograph of a } \\
\text { mosquito/similar } \\
\text { insect that meets } \\
\text { most of the } \\
\text { features of } \\
\text { another species }\end{array}$ & $\begin{array}{l}\text { Reports with } \\
\text { the } \\
\text { photograph of } \\
\text { another } \\
\text { species of } \\
\text { mosquito/ } \\
\text { similar insect }\end{array}$ \\
\hline
\end{tabular}

Figure 2: Diagram of the multi-proxy validation system (top), and categories used for the validation of mosquito reports (bottom). In the middle, a map screenshot and a citizen scientist's picture of a tiger mosquito. 
classification method in the public webmap. However, crowd (if available) and app user validation results are also displayed in the report pop-ups. Reports without pictures are displayed as 'unclassified'.

\section{Lessons from the first year of implementation (2014)}

During the 2014 data collection period (late spring to late winter 2014), almost 7,000 people downloaded Tigatrapp and registered as users (Figure 3). In total, $\sim 2,900$ reports (including mission answers) were sent from $\sim 1,300$ unique user identifiers. Both app download and data collection dynamics were strongly influenced by media appearances. Most reports $(\sim 60 \%)$ were of mosquito sightings, followed by mission answers ( $30 \%)$ and breeding sites $(\sim 10 \%)$. It is not clear why breeding site reports have been so much less frequent than adult mosquito reports. It could be that people are less motivated to report breeding sites than to report adult mosquitoes (which may have just bitten them), or that the complexity of the breeding site concept (e.g. indirect cause-effect due to mosquito life cycles) or the requirement that breeding site reports include photographs makes their reporting more challenging. Whatever the reason, the low number of breeding site reports in 2014 led us to focus the expert validation for that year on only the adult mosquito reports. In contrast, our strategy for 2015 has been to improve breeding site reporting by working with public mosquito management agencies to increase outreach and ensure that breeding site reports lead to tangible results in urban public spaces (see next Section).

Participation in crowd validation ('Tigafotos' project in Crowdcrafting.org) was low ( $\sim 300$ validated photographs out of $\sim 1,200$ ) and heterogeneous in time. Being hosted in a separate platform, it is difficult to compare participation trends to Tigatrapp participation. On one hand, hosting a crowd based photograph validation system on an international crowdsourcing platform has clear benefits in terms of participation, visibility and ease of implementation. On the other hand, this approach has the drawback of relying on participants who are disconnected from the project and its objectives. Crowd engagement in the Tigafotos project might be improved by embedding it also in the AtrapaelTigre.com website (Crowdcrafting.org allows for that), improving the project's design, making it multi-lingual, and adding other gamification and engagement elements.

Although photographs are the basis for report validation (expert and crowd), photograph attachments are optional for mosquito reports. This allows users to report mosquitoes without having to catch them. However, it may be that this makes it too easy for users to avoid making the effort of taking a picture, even when they are able to. In 2014, only around 30\% of the adult mosquito reports actually included a photograph (Figure 3 ). Of those, expert validation approximately assigned $\sim 40 \%$ to the "Unknown" category, $50 \%$ to 'Confirmed Asian 
tiger mosquito' or 'Possible tiger mosquito', and 10\% to 'Possible other species” or "Other species' (Figure 2). Improving users' skill in taking photographs of mosquitoes might well result in more valuable data by moving reports out of the 'Unknown' category. We are now using Tigatrapp missions and embedded information, social media (Twitter and Facebook), and the project blog to systematically train and encourage users to take more and better pictures. These strategies seem to be improving the fitness for use of data considerably, since the number of adult reports with pictures increased to $60 \%$ (30\% in 2014) and the number of reports that could be classified as "Confirmed Asian tiger mosquito" or "Possible tiger mosquito" in 2015 was 1,700, almost the same as the total number of mosquito reports (with or without pictures) received in 2014 , i.e. $~ 1,740$ (Figure 3). We are also developing quantitative methods to make reports without photographs more useful for scientific and management purposes. For example, by combining responses to the taxonomic survey in each report with knowledge about the user based on the quality and quantity of previous reports, we may better assess the probability that a given report corresponds to an actual tiger mosquito. Other methods, like taxonomic validation of georeferenced mosquitoes sent by post (Kampen et al. 2015) may be explored in the future, and the ultimate goal will be to compare the results of several independent validation methods, along with semi-automated and intelligent algorithms based on prior knowledge.

\section{RESULTS: 1st YEAR OF PROJECT IMPLEMENTATION IN SPAIN}

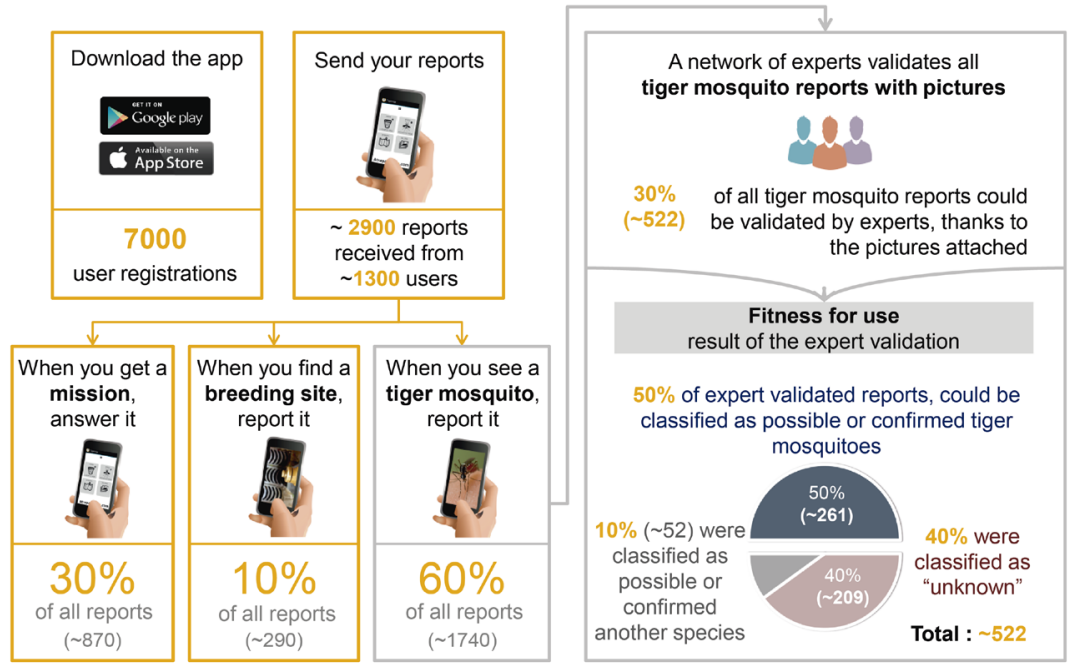

Figure 3: Summary infographic of the results obtained during 2014, the first year of project implementation in Spain (numbers are approximate). 


\section{Integrating citizen science and VGI in the management of invasive species and disease vectors}

Our experience with AtrapaelTigre.com has made it clear that using citizen science to target invasive species or disease vectors requires at least three basic domains of expertise: i) communication and education, ii) surveillance and control and iii) research (Figure 4). These domains and their interrelations acquire a whole new dimension as a consequence of the citizen scientists' involvement and the need to implement control measures that complement current environmental management and public health system polices. Here we discuss how to improve the project in each of these domains, and the main challenges.

\section{Communication and education}

The project has 3 communication and education objectives: 1) spread the word to gain new participants and wider geographic coverage, 2) keep the interest of participants and the media, and 3) inform participants so that they not only provide useful data but also take control actions at places out of the scope of public administrations (e.g. their houses). For instance, by spotting and recognizing breeding sites, citizens become aware of the importance of water removal in their backyards. To accomplish these objectives, the project actively disseminates information through a blog, Facebook and Twitter accounts, press releases, and talks and workshops for different audiences. The project also collaborates with public administrations and private stakeholders and encourages these entities to include information about AtrapaelTigre.com in their own outreach campaigns (e.g. flyers, websites, media appearances). In 2015, we have coordinated communication actions with the Barcelona Public Health Agency (ASPB) and public entities in Valencia and the Canary Islands, amongst others. We are not explicitly assessing trends in public awareness or the population behavior change related to project actions. However, several indicators demonstrate a good performance in terms of communication: media appearances increased each year (see project website for a full list) and sessions in the project website have more than quadrupled between 2014 and 2015, based on estimates from Google Analytics website data between July and December.

From a communication perspective, we have developed a two-fold strategy. We offer a regular stream of new, interesting, and in-depth scientific outreach material related to the targeted species and to other mosquito disease vectors aspects. At the same time, we use project results to demonstrate and explain how citizen scientist participation can help to improve the surveillance and control of the species in the short term. This is a challenge, but of high importance, since most people are likely to participate, not out of scientific interest, but because they are affected by the presence of the species and have a personal interest in its local eradication. 


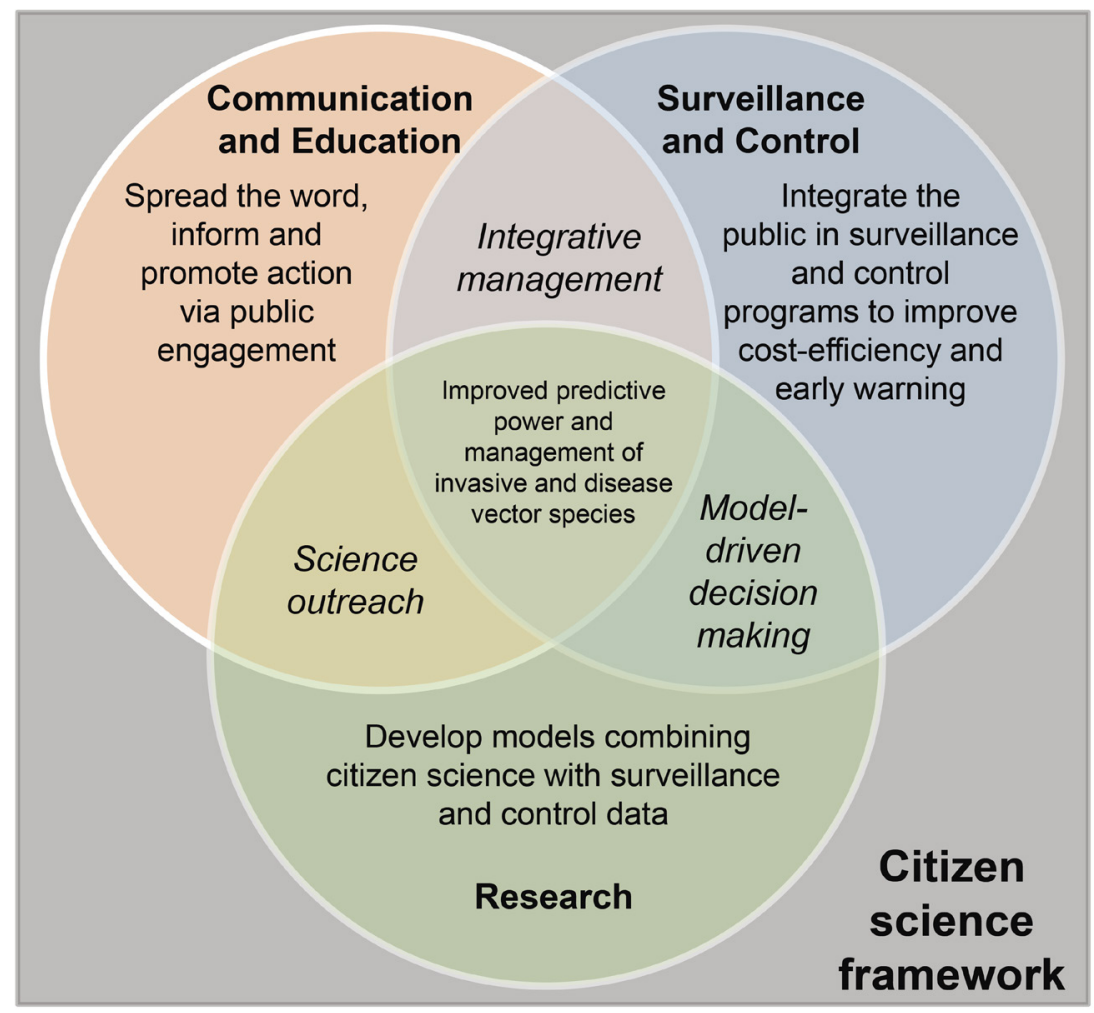

Figure 4: Identified expertise domains for improved predictive power and management of invasive species and disease vectors under a citizen science framework.

\section{Surveillance and control}

Tiger mosquito surveillance and control programs in Spain currently involve a traditional integrative management approach that incorporates communication and education. The citizen science framework (Figure 4) goes beyond this by exploiting new technologies (apps, webmaps, and social media) that enable massive and systematic calls-to-action while making the resulting data immediately available to management services and the general public. It has been demonstrated elsewhere that public participation (even more through the use of new technologies) can advance the detection of an invasive species even 2 years before traditional monitoring programs (Scyphers et al. 2014).

The usefulness of participative frameworks as early warning systems (the primary role of surveillance) is confirmed in the case of AtrapaelTigre.com. By enlisting a large team of citizen scientists while also engaging with the network 
of management agencies and other tiger mosquito stakeholders in Spain, we have been able to gather critical information and pass it to the actors responsible for surveillance in the implicated regions. These actors are then able to decide whether to further investigate and activate relevant environmental and public health protocols. For example, the first-ever report of tiger mosquitoes in Andalusia came from a citizen scientist via Tigatrapp in 2014. After detecting this as a credible alarm, we contacted specialists there, who corroborated the presence of the species in the field (Delacour-Estrella et al. 2014). Similarly, citizen scientists using Tigatrapp were also the first to detect tiger mosquitoes in the Catalan pre-Pyrenees, a discovery confirmed by rural agents and passed through social networks to raise awareness (pers. comm.). Citizen science systems like AtrapaelTigre.com should not be seen as substitutes for active surveillance (e.g. targeted sampling methods) by specialists. The detection of the species in the Basque Country in 2014, for instance, came about only through active surveillance (Delacour-Estrella et al. 2015), demonstrating the extent to which the two approaches are complimentary. Indeed, efficiency of combining passive (e.g. data gathered by the general public) and active surveillance for mosquitoes in Europe is increasingly apparent (Kampen et al. 2015).

Despite their importance, early warnings are only part of the story. There are many regions in Spain where the tiger mosquito has already become established and well known. The challenge for AtrapaelTigre.com in these regions is to build up a participatory system for management and control, reducing the public health risk and improving life quality. To this end, we have contacted actors and stakeholders responsible for control programs in these areas, and developed tools (e.g. interactive web-interfaces) to make citizen science data more accessible and useful for them. This step is costly but it has made the project much more powerful, as the data from citizen scientists can be immediately used to improve management and control in affected areas where this joint collaboration is established. For instance, in 2015, the ASPB incorporated part of its team directly into the AtrapaelTigre.com expert validation system, and it is using citizen science data from the project to improve tiger mosquito control in the city of Barcelona. A similar strategy was followed by stakeholders in the city of Valencia in the same year, and all signs are that this type of involvement is highly beneficial. The numbers of breeding sites' reports have doubled in 2015 (although the total number is still much smaller than for mosquito reports). In Barcelona, 20\% of $\sim 280$ adult and breeding sites' reports received in 2015 in and around the city, were considered useful by the ASPB for management purposes and were incorporated into their already long-lasting Public space surveillance and control program. In Valencia, with a more recent history of surveillance programs starting in $2014,40 \%$ of the detected positive breeding sites in the city were thanks to citizen's reports (breeding sites and adults). Indeed, without discounting the importance of engagement with the citizen scientists themselves, our renewed efforts to communicate with stakeholders are proving crucial for the long-term maintenance of the whole participatory system. 


\section{Research}

Often citizen science is challenged by sampling bias (based, for example, on the distribution of users across territory, or the involvement of restricted social layers) and large variations in the quality of the data (Dickinson et al. 2010). However, similar problems are also present in field data collected by professional scientists (e.g. biodiversity estimates, density estimates of a population). Mosquito surveillance in many European countries often relies on ovitrap networks (networks of traps in which females are prone to lay their eggs). Such trap networks can detect the presence of tiger mosquitoes but do not provide good estimates of abundance, can generate false negatives, can be biased by placement and exposure time, and are generally limited to recently colonized or highly populated areas, leaving large gaps in territorial coverage. An important challenge for AtrapaelTigre.com (and, by extension, to other citizen science projects) is to demonstrate how the combination of citizen scientist data and ovitrap surveillance (Kampen et al. 2015) can improve predictions of the distribution (current and potential), risk factors, and spreading dynamics of the targeted species.

The key is to let the strengths of each approach compensate for the other's weaknesses and to use reliable results from each as a means of cross-calibration. For example, there is now a large amount of ovitrap data available and Tigatrapp data covering the same areas of Spain, as well as Tigatrapp data for areas and times for which ovitrap data is lacking. We are using the ovitrap data in the overlapping areas to calibrate models built from the Tigatrapp data, and we are then using these calibrated models to make estimates about the areas and times for which the ovitrap data is absent. We expect such novel modelling approaches to produce more robust conclusions that contribute to costeffective management strategies.

\section{Conclusions}

Once the infrastructure and basic implementation of a citizen science project has been put in place, attention turns to sustaining long term participation and obtaining sound scientific conclusions from the volunteered data. For projects like AtrapaelTigre.com, that focus on invasive species or disease vectors, participants are often motivated more by management and control goals than scientific interest, making it important to work closely with environmental and public health agencies and related stakeholders. At the same time, the project must be built on a solid scientific foundation and this requires novel approaches to data validation and analysis.

AtrapaelTigre.com uses three independent methods for validating citizen scientist reports, as well as a background location feature that makes it possible to estimate sampling effort and correct bias. Moreover, Tigatrapp's passive citizen 
scientist data is combined with active ovitrap surveillance, with each data type complementing the other and allowing for cross-calibration.

Our 2014 and 2015 results suggest that these methods are promising, but still must be improved. We hope to increase more the quality and quantity of citizen scientists' photographs and to develop new quantitative methods for assessing the reliability of reports without attached photographs. We also hope to improve the multi-proxy validation system, as each of the three validation methods has its own set of drawbacks: the crowd and app-user validation methods are capable of handling massive data but are prone to error, while expert validation appears more accurate but is more costly and better suited to limited quantities of data. The goal is to find ways for the expert validation of a manageable part of the data to inform and improve the crowd and app-user validation for the rest. Finally, another important step will be to formally frame all of these methods in a semi-automated, intelligent alert system that incorporates prior knowledge and directs interesting findings back to stakeholders and the general public.

\section{Final note: from AtrapaelTigre.com to Mosquito Alert}

On February 2016, at the time of editing this manuscript, the project incorporated a new target species, the yellow fever mosquito (Aedes aegypti) and changed name accordingly: from AtrapaelTigre.com, specific for the tiger mosquito, to Mosquito Alert (available at www.mosquitoalert.com). The yellow fever mosquito has a similar appearance and behavior to the Asian tiger mosquito and is currently considered the primary vector of yellow fever, chikungunya, dengue and Zika viruses (ECDC 2016), having raised a lot of international concern in the recent American Zika outbreak (Kindhauser et al. 2016). The species was historically present in Spain, and could be reintroduced again through the island of Madeira, where it is known to be the cause of a dengue outbreak in 2012 (ECDC 2016). In this sense, the incorporation of this new species for the project in Spain is relevant in public health terms and follows primarily an early warning system strategy.

\section{Acknowledgments}

AtrapaelTigre.com has been funded by the Spanish Foundation for Science and Technology-Ministry of Economy and Competitiveness (FCT-12-3730, FCT13-7019), Bloom, Lokímica and Plan Estatal I+D+I. (CGL2013-43139-R), in collaboration with "la Caixa" Banking Foundation. The research leading to these results has received also funding from RecerCaixa. We want to thank all of the participants, students, volunteers, and professionals who have made the project possible. We would like to thank the Barcelona Public Health 
Agency, the Valencia City Council and the company lokímica for assessing the performance of AtrapaelTigre.com in their surveillance and control programs. The images shown in the Tigatrapp screenshot in Figure 1 include Mosquito, designed by Monika Ciapala, Water, designed by Alessandro Suraci, Pot, designed by factor[e] design initiative, Location, designed by Alex Auda Samora, Map designed by Jonathan Higley, and Images designed by Simon Henrotte, all obtained from the Noun Project under the Creative Commons Attribution (CC BY 3.0) license. Figure 3 was done with the help of the project communication team: A. Ramon, M. Torres and J. L. Ordóñez.

\section{References}

Alarcón-Elbal, P. M., Delacour-Estrella, S., Ruiz-Arrondo, I., Collantes, F., Delgado, J. A. Morales-Bueno, J., Sánchez-López, P. F., Amela, C., Sierra-Moros, J. M., Molina, R., \& Lucientes, J. 2014. Updated distribution of Aedes albopictus (Diptera: Culicidae) in Spain: new findings in the mainland Spanish Levante, 2013. Memórias do Instituto Oswaldo Cruz, 109(6): 782-786. DOI: http://dx.doi.org/10.1590/0074-0276140214

Aranda, C., Eritja, R., \& Roiz, D. 2006. First record and establishment of the mosquito Aedes albopictus in Spain. Medical and Veterinary Entomology, 20(1): 150-152. DOI: http://dx.doi.org/10.1111/j.1365-2915.2006.00605.x

Dickinson, J. L., Zuckerberg, B., \& Bonter, D. N. 2010. Citizen science as an ecological research tool: Challenges and benefits. Annual Review of Ecology, Evolution, and Systematics, 41: 149-72. DOI: http://dx.doi.org/10.1146/ annurev-ecolsys-102209-144636

Kampen, H., Medlock, J. M., Vaux, A. G. C., Koenraadt, C. J. M., van Vliet, A. J. H., Bartumeus, F., Oltra, A., Sousa, C. A., Chouin, S., \& Werner, D. 2015. Approaches to passive mosquito surveillance in the EU. Parasites \& Vectors, 8: 9. DOI: http://dx.doi.org/10.1186/s13071-014-0604-5

Delacour-Estrella, S., Collantes, F., Ruiz-Arrondo, I., Alarcón-Elbal, P. M., Delgado, J. A., Eritja, R., Bartumeus, F., Oltra, A., Palmer, J. R. B., \& Lucientes, J. 2014. Primera cita de mosquito tigre, Aedes albopictus (Diptera, Culicidae), para Andalucía y primera corroboración de los datos de la aplicación Tigatrapp. Anales de Biología, 36: 93-96. DOI: http://dx.doi.org/10.6018/ analesbio.36.16

Delacour-Estrella, S., Barandika, J. F., García-Pérez, A. L., Collantes, F., RuizArrondo, I., Alarcón-Elbal, P. M., Bengoa, M., Delgado, J. A., Juste, R. A., Molina, R., \& Lucientes, J. 2015. Detección temprana de mosquito tigre, Aedes albopictus (Skuse, 1894), en el País Vasco (España). Anales de Biología, 37: 25-30. DOI: http://dx.doi.org/10.6018/analesbio.37

European Center for Disease Prevention and Control Aedes aegypti. Available at: http://ecdc.europa.eu/en/healthtopics/vectors/mosquitoes/Pages/aedesaegypti.aspx [Last accessed 16 March 2016]. 
Goodchild, M. F. 2007. Citizens as Sensors: The World of Volunteered Geography. GeoJournal, 69(4): 211-221. DOI: http://dx.doi.org/10.1007/s10708007-9111-y

Hawley, W. A. 1988. The biology of Aedes albopictus. Journal of the American Mosquito Control Association, (Supplement 1): 1-39

Kindhauser, M. K., Allen, T., Frank, V., Santhana, R., \& Dye, C. 2016. Zika: the origin and spread of a mosquito-borne virus. Bulletin of the World Health Organization. DOI: http://dx.doi.org/10.2471/BLT.16.171082

Paupy, C., Delatte, H., Bagny, L., Corbel, V., \& Fontenille, D. 2009. Aedes albopictus, an arbovirus vector: from the darkness to the light. Microbes and Infection, 11(14-16): 1177-1185. DOI: http://dx.doi.org/10.1016/j. micinf.2009.05.005

Roiz, D., Eritja, R., Melero-Alcibar, R., Molina, R., Maruès, E., Ruiz, S., Escosa, R., Aranda, C., \& Lucientes, J. 2007. Distribucion de Aedes (Stegomyia) albopictus (Skuse, 1894) (Diptera, Cuclicidae) en España. Boletín Sociedad Entomológica Aragonesa, 40: 523-526

Scyphers, S. B., Powers, S. P., Adkins, J. L., Drymon, J. M., Martin, C. W., Schobernd, Z. H., Schofield, P. J., Shipp, R. L., \& Switzer, T. S. 2014. The role of citizens in detecting and responding to a rapid marine invasion. Conservation Letters, 8(4): 242-250. DOI: http://dx.doi.org/10.1111/conl.12127 